\title{
Isolated Adrenocorticotropic Hormone Deficiency Caused by Camrelizumab in a Patient with Lung Sarcoma: A Case Report and Literature Review
}

\section{Wen Huang}

The First Affiliated Hospital of Shandong First Medical University \& Shandong Provincial Qianfoshan Hospital

\section{Saran Feng ( $\nabla$ fengqiongxin@163.com )}

The First Affiliated Hospital of Shandong First Medical University \& Shandong Provincial Qianfoshan Hospital

\section{Case report}

Keywords: isolated adrenocorticotropic hormone deficiency, camrelizumab, lung sarcoma, hyponatremia

Posted Date: November 22nd, 2021

DOI: https://doi.org/10.21203/rs.3.rs-1081360/v1

License: (c) (i) This work is licensed under a Creative Commons Attribution 4.0 International License. Read Full License 


\section{Abstract \\ Background}

Immune checkpoint inhibitors significantly increase survival in some malignant tumors, but they also lead to a variety of immune-related adverse events that affect multiple organs, including the liver, lung and endocrine system. Isolated adrenocorticotropic hormone (ACTH) deficiency (IAD) is a rare pituitary disorder characterized by secondary adrenal insufficiency with low or absent cortisol production but the normal secretion of pituitary hormones other than ACTH. IAD caused by atezolizumab, pembrolizumab and nivolumab therapy had been reported, but none is reported in camrelizumab, a new immune checkpoint inhibitor (ICl). Clinicians may not be aware of this unusual side effect.

\section{Case presentation:}

Here, we describe a 70-year-old male patient who had lung sarcoma and was treated with camrelizumab. After nine months of treatment, he developed immune-related pneumonia and hepatitis associated with camrelizumab. The patient discontinued the drug and recovered soon after administration of glucocorticoids. But he subsequently appeared severe hyponatremia, and was diagnosed with late-onset IAD induced by camrelizumab. His symptoms alleviated after glucocorticoid replacement therapy.

\section{Conclusion}

Regular monitoring serum sodium levels and testing endocrine function should be beneficial for cancer patients not only during the treatment of camrelizumab but also after discontinuation of the ICI. Clinicians should consider IAD induced by ICls when hyponatremia occurs in the patients.

\section{Background}

With the development of medicine, cancer immunotherapy has made rapid progress. It has become another important antitumor method besides surgery, radiotherapy and chemotherapy. As a new type of antitumor medications, immune checkpoint inhibitors (ICls) attract more and more attention. It targets cytotoxic T-lymphocyte associated antigen 4, programmed cell death receptor 1 (PD-1), and programmed cell death ligand 1 (PD-L1) to enhance autoimmune response and then exert its antitumor effect [1,2]. However, with rapid promotion of clinical trials and wide applications of ICls, many adverse reactions are being realized. The adverse reactions related to the ICls action mechanism are called immune-related adverse events (irAEs). irAEs can occur in any tissue or organ, including skin, lung, hepatitis and rash [35]. ICls also causes endocrine dysfunctions [6]. The common endocrine related adverse events are thyroid dysfunctions and hypophysitis [6-8], while isolated adrenocorticotropic hormone (ACTH) deficiency (IAD) is rare. IAD has occult symptoms, easy to be misdiagnosed and neglected. Carelizumab (trade name: Erica) is a new type self-developed PD-1 inhibitor in China [9]. It was officially approved to market in May 
2019. The drug has been used to treat many types of malignant tumors. We hereby report a patient with lung cancer who developed severe hyponatremia due to IAD associated with camrelizumab. Meanwhile, we review the characteristics of IAD in order to provide some references for clinical work.

\section{Case Presentation}

The patient was a 70-year-old male. His primary pulmonary nodule was discovered during his regular check-up in June 2019. Through computerized tomography (CT) guided biopsy and histopathology, the patient was diagnosed with lung sarcoma and experienced lung local microwave ablation. He had no special family or psycho-social history. The patient had been treated with camrelizumab monotherapy (200 mg every 2 weeks) since July 2019. Serum electrolytes were normal several times during the treatment. After nine months of treatment, he started to frequently complain of fever, obvious fatigue, anorexia, nausea, vomiting and abdominal distension. He was admitted to our hospital in April 2020. Blood biochemical examination showed hyponatremia [ $\mathrm{Na} 131 \mathrm{mmol} / \mathrm{L}$ (137-147 mmol/L)], liver function damage [ALT 139.9 U/L (9-50 U/L), AST 135.1 U/L (15-40 U/L), GGT780.6 U/L (10-60 U/L), TBIL 73.2 $\mu \mathrm{mol} / \mathrm{L}(5-24 \mu \mathrm{mol} / \mathrm{L})]$, increased leucocytes and neutrophils [WBC $26.14 \times 10^{9} / \mathrm{L}\left(3.5-9.5 \times 10^{9} / \mathrm{L}\right), \mathrm{N}$ $\left.20.13 \times 10^{9} / \mathrm{L}\left(1.8-6.3 \times 10^{9} / \mathrm{L}\right)\right]$. At that time, plasma ACTH, cortisol level and thyroid function were normal. Chest CT showed multifocal flocculent and cord-like high-density shadows in both lungs and bilateral pleural effusion (figure 1A). We diagnosed the patient with immune-related pneumonia and hepatitis associated with camrelizumab and then discontinued the drug. Methylprednisolone $(120 \mathrm{mg} / \mathrm{day})$ was administered to the patient intravenously immediately. Ten days post-infusion, his general condition was rapidly improved. The lesions in the lungs and the pleural effusion almost went away (figure 1B). One month of glucocorticoids therapy later, the hepatic enzyme levels returned to normal range. The patient was discharged from the hospital and gradually terminated glucocorticoids in July 2020.

Since November 2020, the patient often developed slight fever, anorexia, nausea, vomiting and general weakness. He was admitted to our hospital again because these symptoms worsened in February 2021. He had been losing weight (over 10 pounds in two months). Laboratory examinations revealed severe hyponatremia ( $\mathrm{Na} 116 \mathrm{mmol} / \mathrm{L}$ ), but his plasma glucose, liver and renal functions remained normal.

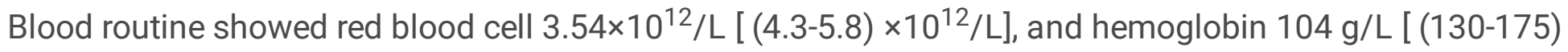
$\mathrm{g} / \mathrm{L}]$. The hormonal assessment showed that plasma ACTH and cortisol levels at each time point were markedly lower than the normal limits (Table 1). Plasma renin activity (PRA) and aldosterone concentration (PAC) were normal. The levels of other anterior pituitary hormones were almost within normal limits (Table 1). Magnetic resonance imaging (MRI) of the pituitary gland showed no abnormalities. Ultrasonography of the thyroid gland and CT examination of the adrenal glands all revealed normal. Based on these findings, he was diagnosed with IAD associated with hyponatremia. His symptoms and hyponatremia were dramatically improved after replacement with hydrocortisone $20 \mathrm{mg}$, orally once a day. The patient was followed up at our outpatient clinic after discharge. Three months later, he had normal serum level of sodium and other pituitary hormone levels, except low levels of ACTH and 
cortisol. He has been well on corticosteroid replacement therapy up to now, with no recurrence of hyponatremia.

Table 1 Hormone assessment showing decreased ACTH and cortisol, elevated TSH and TgAb, elevated prolactin

\begin{tabular}{llll} 
Cortisol circadian rhythm & 8 a.m. & 4 p.m. & 12 p.m. \\
\hline Cortisol (normal range: $172-497)(\mathrm{nmol} / \mathrm{L})$ & 32.65 & 30.85 & 28.16 \\
\hline ACTH (normal range: $5-60)(\mathrm{pg} / \mathrm{ml})$ & 9.55 & 9.78 & 7.44 \\
\hline TSH (normal range: $0.27-4.2)(\mu \mathrm{lU} / \mathrm{mL})$ & 10.05 & & \\
\hline Free T3 (normal range: $3.1-6.8)(\mathrm{pmol} / \mathrm{L})$ & 6.26 & & \\
\hline Free T4 (normal range: $12-22)(\mathrm{pmol} / \mathrm{L})$ & 17.63 & & \\
\hline TPOAb (normal range: $0-34)(\mathrm{IU} / \mathrm{mL})$ & 8.98 & & \\
\hline TgAb (normal range: $0-115)(\mathrm{IU} / \mathrm{mL})$ & 990.30 & & \\
\hline FSH (normal range: $1.5-12.4)(\mathrm{IU} / \mathrm{L})$ & 10.83 & & \\
\hline LH (normal range: $1.7-8.6)(\mathrm{IU} / \mathrm{L})$ & 8.26 & & \\
\hline Prolactin (normal range: $4.04-15.2)(\mathrm{ng} / \mathrm{ml})$ & 29.72 & \\
\hline Testosterone (normal range: $2.8-8.0)(\mathrm{ng} / \mathrm{ml})$ & 5.52 & \\
\hline GH (normal range: $0.06-5.0)(\mathrm{ng} / \mathrm{ml})$ & 1.05 & \\
\hline IGF-1 (normal range: $60-350)(\mu \mathrm{g} / \mathrm{ml})$ & 82.10 & \\
\hline Plasma renin (normal range: $4.4-46.1)(\mathrm{ulU} / \mathrm{ml})$ & 8.6 & \\
\hline Aldosterone (normal range: $3.0-35.3)(\mathrm{ng} / \mathrm{L})$ & 17.46 & \\
\hline
\end{tabular}

ACTH, adrenocorticotropic hormone; TSH, thyroid-stimulating hormone; TPOAb, anti-thyroperoxidase antibody; $\mathrm{TgAb}$, anti-thyroglobulin antibody; FSH, follicle stimulating hormone; $\mathrm{LH}$, luteinizing hormone; GH, growth hormone; IGF-1, insulin-like growth factor 1

\section{Discussion}

Camrelizumab, as a new type PD-1 inhibitor, is increasingly used in clinical anti-tumor therapy. With its wide application, people gradually realize its immune-related adverse events. Here we report a rare case that camrelizumab induced immune-related pneumonia and hepatitis, subsequent IAD. Nine months after the administration of camrelizumab, the cancer patient developed anorexia, elevated transaminase and bilirubin, fever, and diffuse plaque infiltration in the lung fields. He was diagnosed with immune-related pneumonia and hepatitis. After promptly treated with methylprednisolone, his symptoms alleviated. His 
pulmonary inflammation was absorbed and his liver function indexes returned to normal in a short time. It demonstrated that glucocorticoids therapy was effective. His plasma ACTH, cortisol levels and thyroid functions remained within the normal ranges during that period. Hyponatremia in the patient was considered to be caused by poor anorexia and low food intake.

During the treatment of PD-1 inhibitors, the most prevalent endocrine irAEs is thyroid dysfunctions [7], followed by hypophysitis $[6,8]$. Thyroid dysfunctions are mainly characterized by asymptomatic thyroiditis. The frequencies of hyperthyroidism and hypothyroidism are $3.1 \%$ and $7.2 \%$ [10], respectively. The patient in the case presented subclinical hypothyroidism with the abnormal high levels of TSH and $\mathrm{TgAb}$. It seemed reasonable to diagnose him with immune-related thyroiditis caused by ICl. Hypopthysitis often occurs in men over 60 years old $[11,12]$. The clinical symptoms of hypophysitis are usually atypical, such as headache and fatigue [13]. It is often accompanied by multiple hormone deficiency, including TSH (86\% 100\%), gonadotropin (85\% 100\%), ACTH (50\% 73\%) [14-17]. 47\% 50\% of the cases may be accompanied by hyponatremia but diabetes insipidus is rare $[6,15,18]$. Pituitary MRI is very sensitive imaging diagnostic examination, showing that pituitary gland increases moderately $(30 \% \sim 100 \%)[17,19]$. But IAD induced by ICls is rare. IAD is secondary adrenal insufficiency (AI), with low or absent cortisol production, but all other pituitary hormones secreted normally except ACTH. Unlike autoimmune hypophysitis, where a mild to moderate diffuse pituitary enlargement seen in all patients and these morphological alterations can even precede the clinical diagnosis in half of the cases [20], the pituitary morphology on MRI in IAD induced by ICls is usually normal. It is possible that the involvement of a single anterior pituitary cell type, in this case corticotroph, is insufficient to develop anatomical alterations that can be seen in imaging tests [21]. In addition, although Al is not associated with mineralocorticoid deficiency, hyponatremia often occurs in IAD as a result of reduced glomerular filtration rate, increased antidiuretic hormone secret and concomitant hypothyroidism [22]. Patients with IAD usually present with non-specific manifestation, so it may be misinterpreted or underdiagnosed. Once cancer patients treated with ICls develop non-specific symptoms along with hyponatremia, the diagnosis of IAD should be considered [20,22, 23]. In this case, the patient was treated with camrelizumab. 1.5 years later, he developed fatigue, loss of weight and anorexia, accompanied by severe hyponatremia. Combined with his medication history, we took IAD induced by ICls into account. Hormonal profiles of the patient demonstrated hypocortisolemia, low ACTH levels, normal function of other pituitary axes and normal plasma aldosterone levels. These results were consistent with the diagnosis of IAD.

Previous studies have described IAD associated with atezolizumab, pembrolizumab and nivolumab therapy [24-28], most of which have occurred within a few months of initiating ICls therapy [11]. In this case, it was seven months after discontinuation of camrelizumab that the patient occurred IAD. Therefore, cancer patients treated with ICls require regular follow-up tests of hyponatremia and endocrine functions not only during the treatment but also after discontinuation in order to find IAD in time.

Once IAD occurs, glucocorticoid replacement therapy can early improve the clinical symptoms. After given $20 \mathrm{mg}$ hydrocortisone replacement therapy, the patient's symptoms soon alleviated. The increase of 
prolactin and the decrease of hemoglobin in the patient were considered to be caused by the lack of glucocorticoids [29]. The indexes returned to normal after glucocorticoid supplement therapy.

\section{Conclusion}

Hyponatremia is a powerful predictor of IAD. Clinicians should consider the diagnosis of IAD when cancer patients treated with the $\mathrm{ICl}$ develops significant asthenia or unexpected non-specific symptoms along with hyponatremia. In additional, regular follow-up tests of hyponatremia and endocrine function are all essential for these patients not only during the treatment of camrelizumab but also after discontinuing the ICl.

\section{Abbreviations}

IAD: isolated adrenocorticotropic hormone deficiency; Al: adrenal insufficiency; ICls: immune checkpoint inhibitors; PD-1: programmed cell death receptor 1; PD-L1: programmed cell death ligand 1; irAEs: immune-related adverse events; $\mathrm{CT}$ : computed tomographic; MRI: magnetic resonance imaging; $\mathrm{ACTH}$ : adrenocorticotropic hormone; TSH: thyroidstimulating hormone; TPOAb: anti-thyroperoxidase antibody; TgAb: anti-thyroglobulin antibody; FSH: follicle stimulating hormone; LH: luteinizing hormone; GH: growth hormone; IGF-1: insulin-like growth factor 1; PRA: plasma renin activity; PAC: aldosterone concentration; TRH: thyrotropin-releasing hormone

\section{Declarations}

\section{Acknowledgements}

Not applicable.

\section{Funding}

Not applicable.

\section{Authors' contributions}

Wen Huang is the attending physician of this patient.Wen Huang and Saran Feng are involved in composition and revision of the manuscript. All authors have read and approved the manuscript.

\section{Availability of data and materials}

The datasets used during the current study are available from the corresponding author on reasonable request.

\section{Ethical approval and consent to participate}

Not applicable 


\section{Consent for publication}

Written informed consent have been obtained from the patient for publication of this case report and any accompanying images. A copy can be made available upon request.

\section{Competing interests}

The authors declare that there is no competing interests between them.

\section{References}

1. Topalian SL, Hodi FS, Brahmer JR, Gettinger SN, Smith DC, McDermott DF, Powderly JD, Carvajal RD, Sosman JA, Atkins MB et al: Safety, activity, and immune correlates of anti-PD-1 antibody in cancer. N Engl J Med 2012, 366(26):2443-2454.

2. Pennock GK, Chow LQ: The Evolving Role of Immune Checkpoint Inhibitors in Cancer Treatment. Oncologist 2015, 20(7):812-822.

3. Kahler KC, Hassel JC, Heinzerling L, Loquai C, Mossner R, Ugurel S, Zimmer L, Gutzmer R, Cutaneous Side Effects" Committee of the Work Group Dermatological O: Management of side effects of immune checkpoint blockade by anti-CTLA-4 and anti-PD-1 antibodies in metastatic melanoma. $J$ Dtsch Dermatol Ges2016, 14(7):662-681.

4. Michot JM, Bigenwald C, Champiat S, Collins M, Carbonnel F, Postel-Vinay S, Berdelou A, Varga A, Bahleda R, Hollebecque A et al: Immune-related adverse events with immune checkpoint blockade: a comprehensive review. Eur J Cancer 2016, 54:139-148.

5. Puzanov I, Diab A, Abdallah K, Bingham CO, 3rd, Brogdon C, Dadu R, Hamad L, Kim S, Lacouture ME, LeBoeuf NR et al: Managing toxicities associated with immune checkpoint inhibitors: consensus recommendations from the Society for Immunotherapy of Cancer (SITC) Toxicity Management Working Group. J Immunother Cancer 2017, 5(1):95.

6. Byun DJ, Wolchok JD, Rosenberg LM, Girotra M: Cancer immunotherapy - immune checkpoint blockade and associated endocrinopathies. Nat RevEndocrino/ 2017, 13(4):195-207.

7. Narita T, Oiso N, Taketomo Y, Okahashi K, Yamauchi K, Sato M, Uchida S, Matsuda H, Kawada A: Serological aggravation of autoimmune thyroid disease in two cases receiving nivolumab. $J$ Dermatol 2016, 43(2):210-214.

8. Faje A: Hypophysitis: Evaluation and Management. Clin Diabetes Endocrinol 2016, 2:15.

9. Markham A, Keam SJ: Camrelizumab: First Global Approval. Drugs 2019, 79(12):1355-1361.

10. Barroso-Sousa R, Barry WT, Garrido-Castro AC, Hodi FS, Min L, Krop IE, Tolaney SM: Incidence of Endocrine Dysfunction Following the Use of Different Immune Checkpoint Inhibitor Regimens: A Systematic Review and Meta-analysis. JAMA Oncol 2018, 4(2):173-182.

11. Min L, Hodi FS, Giobbie-Hurder A, Ott PA, Luke JJ, Donahue H, Davis M, Carroll RS, Kaiser UB: Systemic high-dose corticosteroid treatment does not improve the outcome of ipilimumab-related 
hypophysitis: a retrospective cohort study. Clin Cancer Res 2015, 21(4):749-755.

12. Faje A: Immunotherapy and hypophysitis: clinical presentation, treatment, and biologic insights. Pituitary 2016, 19(1):82-92.

13. Faje AT, Sullivan R, Lawrence D, Tritos NA, Fadden R, Klibanski A, Nachtigall L: Ipilimumab-induced hypophysitis: a detailed longitudinal analysis in a large cohort of patients with metastatic melanoma. J Clin Endocrinol Metab2014, 99(11):4078-4085.

14. Barroso-Sousa R, Ott PA, Hodi FS, Kaiser UB, Tolaney SM, Min L: Endocrine dysfunction induced by immune checkpoint inhibitors: Practical recommendations for diagnosis and clinical management. Cancer 2018, 124(6):1111-1121.

15. Scott ES, Long GV, Guminski A, Clifton-Bligh RJ, Menzies AM, Tsang VH: The spectrum, incidence, kinetics and management of endocrinopathies with immune checkpoint inhibitors for metastatic melanoma. Eur J Endocrinol 2018, 178(2):173-180.

16. Chang LS, Barroso-Sousa R, Tolaney SM, Hodi FS, Kaiser UB, Min L: Endocrine Toxicity of Cancer Immunotherapy Targeting Immune Checkpoints. Endocr Rev 2019, 40(1):17-65.

17. Castinetti F, Albarel F, Archambeaud F, Bertherat J, Bouillet B, Buffier P, Briet C, Cariou B, Caron P, Chabre $O$ et al: French Endocrine Society Guidance on endocrine side effects of immunotherapy. EndocrRelat Cancer 2019, 26(2):G1-G18.

18. Zhao C, Tella SH, Del Rivero J, Kommalapati A, Ebenuwa I, Gulley J, Strauss J, Brownell I: Anti-PD-L1 Treatment Induced Central Diabetes Insipidus. J Clin Endocrinol Metab2018, 103(2):365-369.

19. Joshi MN, Whitelaw BC, Palomar MT, Wu Y, Carroll PV: Immune checkpoint inhibitor-related hypophysitis and endocrine dysfunction: clinical review. Clin

20. Endocrinol (Oxf) 2016, 85(3):331-339.

21. Faje AT, Sullivan R, Lawrence D, Tritos NA, Fadden R, Klibanski A, Nachtigall L: Ipilimumab-induced hypophysitis: a detailed longitudinal analysis in a large cohort of patients with metastatic melanoma. J Clin Endocrinol Metab 2014, 99(11):4078-4085.

22. Iglesias P, Sanchez JC, Diez JJ: Isolated ACTH deficiency induced by cancer immunotherapy: a systematic review. Pituitary 2021, 24(4):630-643.

23. Andrioli M, Pecori Giraldi F, Cavagnini F: Isolated corticotrophin deficiency. Pituitary 2006, 9(4):289295.

24. Komatsu T, Ohara N, Hirota N, Yoneoka Y, Tani T, Terajima K, Ozawa T, Sone H: Isolated Adrenocorticotropic Hormone Deficiency Presenting with Severe Hyponatremia and Rhabdomyolysis: A Case Report and Literature Review. Am J Case Rep 2019, 20:1857-1863.

25. Ohara N, Ohashi K, Fujisaki T, Oda C, Ikeda Y, Yoneoka Y, Hashimoto T, Hasegawa G, Suzuki K, Takada T: Isolated Adrenocorticotropin Deficiency due to Nivolumab-induced Hypophysitis in a Patient with Advanced Lung Adenocarcinoma: A Case Report and Literature Review. Intern Med 2018, 57(4):527-535. 
26. Narahira A, Yanagi T, Cho KY, Nakamura A, Miyoshi H, Hata H, Imafuku K, Kitamura S, Shimizu H: Isolated adrenocorticotropic hormone deficiency associated with nivolumab therapy. J Dermatol 2017, 44(4):e70.

27. Kitajima K, Ashida K, Wada N, Suetsugu R, Takeichi Y, Sakamoto S, Uchi H, Matsushima T, Shiratsuchi M, Ohnaka $\mathrm{K}$ et al: Isolated ACTH deficiency probably induced by autoimmune-related mechanism evoked with nivolumab.

28. Jpn J Clin Oncol 2017, 47(5):463-466.

29. Kanie K, Iguchi G, Bando H, Fujita Y, Odake Y, Yoshida K, Matsumoto R, Fukuoka H, Ogawa W, Takahashi Y: Two Cases of Atezolizumab-Induced Hypophysitis. J Endocr Soc 2018, 2(1):91-95.

30. Ariyasu R, Horiike A, Yoshizawa T, Dotsu Y, Koyama J, Saiki M, Sonoda T,

31. Nishikawa S, Kitazono S, Yanagitani $\mathrm{N}$ et al: Adrenal Insufficiency Related to Anti-Programmed Death-1 Therapy. Anticancer Res 2017, 37(8):4229-4232.

32. Shibutani Y: Prolactin dynamics in a patient with isolated ACTH deficiency accompanied by hyperprolactinemia. Am J Med Sci 1988, 295(2):140-143

\section{Figures}
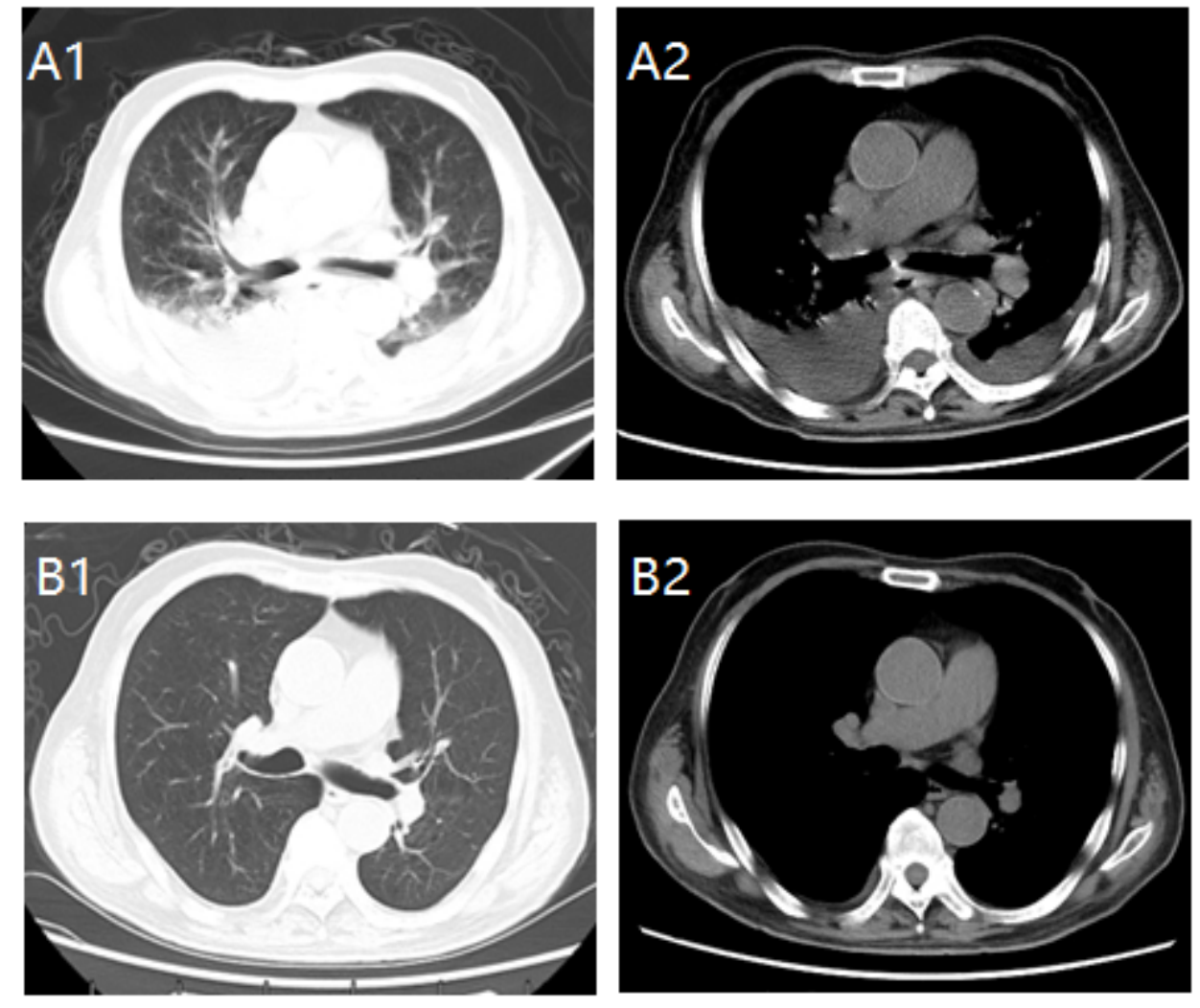

Figure 1 
Chest CT scan before and after therapy with glucocorticoids. (A1, A2) Chest CT reveals that multifocal flocculent and cord-like high-density shadows in both lungs and bilateral pleural effusion before therapy with glucocorticoids. (B1, B2) Chest CT reveals that inflammatory lesions and pleural effusion were almost completely absorbed after therapy with glucocorticoids.

\section{Supplementary Files}

This is a list of supplementary files associated with this preprint. Click to download.

- carechecklist.jpg 